\title{
Protection originale du port de Nuisement sur le lac du Der
}

\author{
Jean-Marc Beynet $^{(\mathrm{a})}$, Jean Bougis ${ }^{(\mathrm{b})}$, Thierry Cherrière ${ }^{(\mathrm{c})}$, \\ Michel Chignoli $^{(\mathrm{d})}$ et Alex Degaie ${ }^{(\mathrm{e})}$
}

\author{
${ }^{(a)}$ Directeur de projet, BRL Ingénierie, 1105 Avenue P. Mendès France, 30001 Nîmes Cedex, \\ Tél. : 04.66.87.51.14, jean-marc.beynet@brl.fr

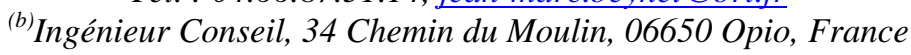 \\ Tél. : 04.93.77.74.22,jbougis@aws.fr \\ ${ }^{(c)}$ Directeur Général, Syndicat du Der, Maison du Lac, 51290 Giffaumont-Champaubert, \\ Tél. : 03.26.72.62.87, syndicat.der@free.fr \\ (d)Ingénieur, BRI Ingénierie, 1105 Avenue P. Mendès France, 30001 Nîmes Cedex, \\ Tél. : 04.66.87.50.00, michel.chignoli@brl.fr \\ ${ }^{(e)}$ P.-D.G. Degaie S.A., 97 Route de Bavay, B.P.5, 59138 Pont-sur-Sambre, \\ Tél.: 03.27.53.61.10, contact@degaie.com
}

\section{Résumé :}

Avec une superficie de 5000 ha, le lac du Der-Chantecoq, en région ChampagneArdennes, est la plus grande réserve artificielle d'Europe. Dédié au soutien des étiages et à l'écrêtement des crues de la Marne, il est aussi utilisé pour la plaisance. Le Syndicat d'Aménagement Touristique du Lac du Der a mis à profit la vidange décennale 2003-2004 pour entreprendre d'importants travaux de réhabilitation sur les ports de plaisance de Nemours et de Nuisement. Les contraintes hydrodynamiques, géotechniques et les délais impartis ont conduit à choisir les atténuateurs semi-submersibles ASB-3000 pour protéger l'entrée du port de Nuisement. Les conditions de ce choix sont exposées et analysées.

\section{$\underline{\text { Abstract: }}$}

With an area of 5000 ha, the lake of Der-Chantecoq, in country of Champagne-Ardennes, is the largest artificial water tank in Europe. Dedicated to support the lowest water-level and to cut the swelling of the Marne river, it is used by the pleasure ships. During the 2003-2004, the decennial of emptying of this lake, the Tourist Development Bureau of the lake of Der has undertaken a large rehabilitation works on the harbours of Nemours and Nuisement. Hydrodynamic and geotechnical constraints and the time allowed are drawing to choose the semi-submersibles wave attenuators ASB-3000 to protect the entry of Nuisement harbour. Conditions of this choice are presented and analysed.

Mots-clés : atténuateur houle, brise-lames flottant, ASB, ancrage, pieux.

Keywords: wave attenuator device, floating breakwater, ASB, anchorage, piles.

\section{Introduction}

Situé dans la région Champagne-Ardennes, le lac du Der-Chantecoq est la plus grande réserve artificielle d'Europe avec une superficie de 5000 ha qui lui confère le statut de mer intérieure (loi littoral : loi n86-2 du 3 janvier 1986). Au début des années 70, l'Institution Interdépartementale des Barrages Réservoirs du Bassin de la Seine a implanté ce lac en dérivation de la Marne pour soutenir ses étiages et écrêter ses crues. Le niveau peut varier entre les cotes $127.5 \mathrm{~m}$ et $139.7 \mathrm{~m}$ IGN 69. Le remplissage a lieu du début novembre à la fin 
juin, tandis que la vidange est effectuée du début juillet à la fin octobre. Les hivers froids, le lac peut geler et être recouvert d'une couche de glace pouvant atteindre $0.4 \mathrm{~m}$.

Le lac est également utilisé pour la plaisance et le Syndicat d'Aménagement Touristique du Lac du Der a mis à profit la vidange décennale 2003-2004 pour entreprendre d'importants travaux de réhabilitation sur les deux ports de plaisance de Nemours et de Nuisement. Lors des études de définition (BRLi ${ }^{1}$ ), différentes solutions ont été étudiées et comparées pour améliorer la protection de ces ports.

A Nemours, la configuration du site, bien abrité par la digue existante contre les clapots les plus sévères provenant du Sud-Ouest, a permis d'en compléter la protection contre les clapots plus faibles d'Ouest $\left(H_{\mathrm{s}}<0.7 \mathrm{~m}-T_{\mathrm{p}}<2.6 \mathrm{~s}\right)$ avec deux pannes de 9 et 5 pontons flottants en béton de longueur $L=12.5 \mathrm{~m}$, de largeur $B=3.5 \mathrm{~m}$, et de tirant d'eau $D=0.8 \mathrm{~m}$.

A Nuisement, la configuration du site, exposé à des conditions hydrodynamiques sévères de secteur Sud-Est à Sud-Ouest $\left(H_{\mathrm{s}} \sim 1.3 \mathrm{~m}\right.$ et $\left.T_{\mathrm{p}} \sim 3.8 \mathrm{~s}\right)$ nécessite une protection nettement plus consistante. Pour construire une digue en terre, la profondeur d'eau importante et la nature argileuse des sols imposaient la mise en œuvre de très gros volumes de matériaux et exposaient à des aléas difficilement maîtrisables quant au contrôle de la teneur en eau du sol pendant le compactage. Le recours à une digue était donc difficilement compatible avec la durée des travaux qui devaient forcément s'inscrire dans le cadre de la vidange décennale. Compte tenu de la profondeur et de la nature du sol, l'utilisation de pontons lourds nécessitait une masse linéique importante $(B=7.1 \mathrm{~m}, D=1.9 \mathrm{~m})$ difficilement compatibles avec une raideur suffisante des ancrages. C'est donc une ligne d'atténuateurs semi-submersibles ASB3000 (Bougis et Degaie $^{2}$ ), d'efficacité spécifique bien supérieure, qui a été retenue pour protéger la passe d'entrée du port.

\section{Dimensionnement des atténuateurs}

\subsection{Principe de fonctionnement}

Brise-lames flottant de type dynamique, l'atténuateur semi-submersible utilise les propriétés ondulatoires de la houle pour la réfléchir. Il est constitué d'une "plaque" horizontale surmontée sur toute sa longueur d'un caisson vertical étroit. Assujetti à coulisser verticalement entre deux pieux, ses cinq autres degrés de liberté sont entravés au moyen de deux colliers de guidage par pieu. Sa faible surface de flottaison associée à sa masse relativement importante et la présence de jupes de confinement de l'eau sous sa plaque lui confère une période propre de pilonnement élevée. Sous l'action de la houle incidente diffractée, l'atténuateur oscille verticalement en générant de part et d'autre une onde radiée. Pour des périodes suffisamment petites devant la période propre de pilonnement de l'appareil $(<2 / 3)$, cette onde est en opposition de phase avec l'onde incidente sur l'arrière et en phase avec elle sur l'avant ; l'onde transmise s'en trouve fortement atténuée.

L'efficacité d'un brise-lames est caractérisée par son coefficient de transmission de hauteur $C_{T}$ (rapport de la hauteur de l'onde transmise à la hauteur de l'onde incidente). La figure 1 montre l'évolution du $C_{T}$ des atténuateurs ASB en grande profondeur en fonction du rapport entre la longueur d'onde $\lambda$ et la largeur $B$ de l'ASB (Bougis ${ }^{3}$ ). Le coefficient de transmission de l'énergie $C_{T}^{\prime}$ est le carré de $C_{T}$. 


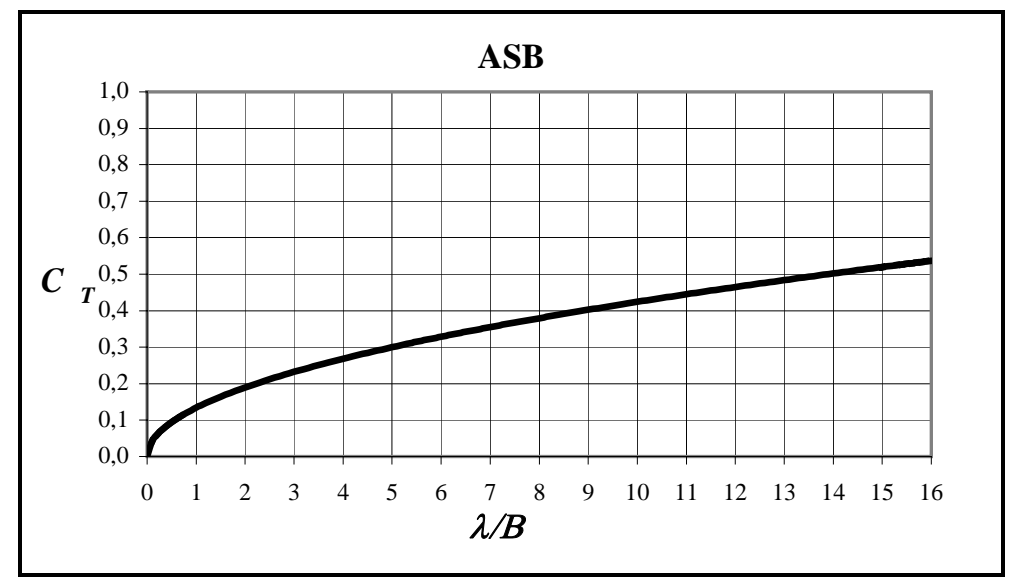

Figure 1: Coefficient de transmission des atténuateurs ASB

\subsection{Dimensionnement des atténuateurs ASB}

Pour obtenir une onde transmise inférieure à $0.50 \mathrm{~m}$ dans les conditions les plus sévères du site $\left(H_{\mathrm{s}} \sim 1.3 \mathrm{~m}\right.$ et $\left.T_{\mathrm{p}} \sim 3.8 \mathrm{~s}\right)$, le coefficient de transmission de hauteur doit être de 0.38 (15\% de transmission de l'énergie). La figure 1 montre que le rapport $\lambda / B$ doit être de l'ordre de 8 . La longueur d'onde d'une houle de $3.8 \mathrm{~s}$ étant de $22.5 \mathrm{~m}$ en grande profondeur, l'appareil doit avoir une largeur supérieure à $2.8 \mathrm{~m}$. Le choix s'est donc porté sur une largeur de $3.0 \mathrm{~m}$ (ASB-3000) dont la période propre est de $8.5 \mathrm{~s}$. Sa période de coupure $\left(C_{T}=0.5\right)$ est de $5.2 \mathrm{~s}$.

Compte tenu de l'éventail important des incidences des ondes $\left( \pm 90^{\circ}\right.$ de part et d'autre de la normale au brise-lames), la longueur $L=12.0 \mathrm{~m}$ des modules a été choisie relativement courte afin que les appareils puissent répondre successivement en fonction de l'instant où ils sont excités par l'onde incidente. Composée de 7 modules espacés de $0.8 \mathrm{~m}$, le brise-lames a une longueur totale de $88.8 \mathrm{~m}$

L'épaisseur de la plaque est de $0.4 \mathrm{~m}$. La hauteur des jupes est de $0.8 \mathrm{~m}$. La hauteur du caisson vertical est de l'ordre de $70 \%$ à $75 \%$ de la hauteur maximale des ondes à atténuer. Pour une hauteur significative de $1.3 \mathrm{~m}$, la hauteur maximale est de l'ordre de $2.2 \mathrm{~m}$. La hauteur du caisson est donc de $1.6 \mathrm{~m}$, répartie de part et d'autre de la surface libre. Le tirant d'eau $D$ est de $2.0 \mathrm{~m}$ et le tirant d'air de $0.8 \mathrm{~m}$. Les deux colliers de guidage sont espacés de $1.7 \mathrm{~m}$ (hauteur du corps de l'appareil). La masse de chaque module est de $25 \mathrm{t}$, dont $7 \mathrm{t}$ de ballast.

\subsection{Efforts dimensionnants}

\subsubsection{Efforts extrêmes}

Le dimensionnement du brise-lames tient compte de trois situations : à flot soumis au vent, au courant et à la houle - à flot soumis au vent, au courant et à une couche de glace de grande superficie libérée par la montée des eaux - au sol lors de la vidange hivernale soumis au vent. Le tableau 1 indique les efforts statiques (dérive sur houle incluse) et dynamiques à considérer dans ces configurations. Les efforts dynamiques ont été décomposés sur les pieux et sur les colliers. Le point $O$ est sur la surface libre. L'axe $O x$ est longitudinal. L'axe $O y$ est transversal. L'axe $O z$ vertical ascendant passe par le centre de gravité $G$. L'incidence de l'onde est définie par l'angle entre $O x$ et sa direction de propagation $\left(90^{\circ}\right.$ pour les houles de travers). Les moments sont réduits en $O$. 
Tableau 1: Efforts dimensionnants pour un module ASB-3000-12 (12 m de long).

\begin{tabular}{|c|r|r|r|r|r|r|r|}
\hline Efforts & \multicolumn{3}{|c|}{ Statiques (module) } & \multicolumn{3}{c|}{ Dynamiques $-H s=1.3 \mathrm{~m}-T_{p}=3$ à 6s } \\
\cline { 2 - 7 } maximaux & A flot & A flot+glace & Hiver au sol & Incidence & module & pieu & collier \\
\hline Fx (kN) & 8.7 & 128.2 & 9.4 & $0^{\circ}$ & 85 & 43 & 66 \\
Fy (kN) & 65.2 & 515.5 & 102.1 & $90^{\circ}$ & 507 & 254 & 769 \\
Fz (kN) & - & - & - & $90^{\circ}$ & 254 & 127 & 129 \\
Mx (kN.m) & 8.5 & 8.5 & 25.5 & $90^{\circ}$ & 2128 & 1064 & - \\
My (kN.m) & 1.5 & 1.5 & 14.8 & $0^{\circ}$ & 152 & 76 & - \\
Mz (kN.m) & - & & - & $45^{\circ}$ & 30 & - & - \\
\hline
\end{tabular}

Les efforts dynamiques sont les efforts extrêmes attendus pour une tempête $H_{\mathrm{s}}=1.3 \mathrm{~m}-$ $T_{\mathrm{s}}=3.8 \mathrm{~s}$. Ils sont statistiquement sensés n'arriver qu'une une seule fois au cours d'une tempête de $6 \mathrm{~h}$. Les efforts significatifs sont deux fois plus faibles. Les efforts sont des enveloppes qui prennent en compte le fondamental et les harmoniques d'ordre 2 ou 3 qui peuvent exister. Ces efforts sont ceux qui s'exerceront réellement ; ils ne tiennent compte d'aucun coefficient de sécurité.

\subsubsection{Périodes propres}

Les périodes propres de flexion de l'ancrage (cavalement, embardée et lacet) doivent être inférieures à la gamme de périodes des lames incidentes. Soit $m$ (25t) la masse d'un module ASB-3000-12, $\mathrm{Cm}_{22}$ son coefficient de masse d'eau ajoutée en embardée (1.3 pour les très petites périodes) et $k$ la raideur de l'ancrage en embardée (rapport de l'effort maximal $F_{\mathrm{m}}$, statique $65 \mathrm{kN}$ et dynamique $507 \mathrm{kN}$ à la déflection maximale $\delta_{m}$ correspondante). La période propre d'embardée s'écrit :

$T=2 \pi \sqrt{\frac{m\left(1+C m_{22}\right)}{k}}=2 \pi \sqrt{\frac{m\left(1+C m_{22}\right) \delta_{m}}{F_{m}}}$

$\mathrm{Si}$, pour le plus haut niveau d'eau, la déflection maximale est de $0.25 \mathrm{~m}$, la période propre d'embardée est inférieure à $1 \mathrm{~s}$. Un raisonnement analogue montre que les périodes propres de cavalement et de lacet le sont également dans les mêmes conditions. Les périodes propres de roulis et tangage sont beaucoup plus petites.

\subsubsection{Tenue à la fatigue}

La tenue aux efforts maximaux étant assurée, les effets de la fatigue doivent être analysés. La loi de Miner stipule que les efforts qui s'inscrivent dans la répétition d'un grand nombre de cycles doivent être évalués à partir de l'ensemble des valeurs statistiques. D'après l'hypothèse de Palmgren-Miner sur le cumul linéaire des endommagements partiels, l'endommagement total peut être évalué à partir de l'analyse spectrale sur la durée de vie de l'ouvrage.

Les conditions limites acceptables pour la fatigue des métaux sont évaluées à partir des courbes de Wöhler qui relient le nombre de cycle admissible $n$ pour un niveau de contrainte donné $\sigma$. Dans le plan $(\log n-\log \sigma)$, ces courbes sont des droites avec un seuil pour les métaux ferreux, et sans seuil pour les métaux non ferreux. C'est là une des raisons fondamentales dans le choix de l'acier et celui de l'élimination de l'aluminium pour de telles applications.

C'est la contrainte équivalente correspondant au critère de von Mises qui a été comparée à la contrainte maximale admissible de Wöhler pour évaluer la résistance de la structure à la fatigue. Ce critère qui correspond au travail de déformation élastique de la structure est bien 
adapté aux métaux. Un coefficient de sécurité de 2.25 a été appliqué sur le nombre de cycles (code de conception Finabel).

\section{Dimensionnement des ancrages sur pieux}

\subsection{Contraintes géotechniques}

Le site d'installation du brise-lames flottant correspond à une ancienne zone d'emprunt dont les matériaux ont servi à la construction des anciennes digues du port. Le sol naturel avait donc été descendu à la cote $126.5 \mathrm{~m}$ et s'était recouvert de $0.8 \mathrm{~m}$ de vase. Pour ce projet, il a été décapé à la cote $126.0 \mathrm{~m}$ puis remblayé à la cote $131.0 \mathrm{~m}$ avec les matériaux argileux sains disponibles à proximité du site.

Avant les travaux, deux sondages pressiométriques ont été réalisés le long de la ligne d'atténuateurs en des points distants de $17.5 \mathrm{~m}$ de chacune des extrémités (tableau 2). Le sol est constitué de marnes grises micacées de caractéristiques géotechniques assez moyennes en surface puis bonnes à excellentes en profondeur avec des pressions limites comprises entre 0.4 et 5 . MPa.

Tableau 2: Caractéristiques du sol sous le brise-lames flottant.

\begin{tabular}{|c|c|c|c|c|c|}
\hline $\begin{array}{c}\text { Cote IGN69 } \\
(\mathrm{m})\end{array}$ & Matériau & \multicolumn{2}{|c|}{ Sondage SP1 } & \multicolumn{2}{c|}{ Sondage SP2 } \\
\cline { 3 - 5 } & & $\mathrm{P}_{1}(\mathrm{MPa})$ & $\mathrm{E}_{\mathrm{M}}(\mathrm{MPa})$ & $\mathrm{P}_{1}(\mathrm{MPa})$ & $\mathrm{E}_{\mathrm{M}}(\mathrm{MPa})$ \\
\hline 131.0 à 140.0 & Eau & - & - & - & - \\
126.0 à 131.0 & Remblai & 0.40 & 5.0 & 0.40 & 5.0 \\
125.5 à 126.0 & Marnes & 1.01 & 14.8 & 0.38 & 1.5 \\
124.5 à 125.5 & Marnes & 1.03 & 14.7 & 0.46 & 2.2 \\
123.5 à 124.5 & Marnes & 1.03 & 10.4 & 2.04 & 22.3 \\
122.5 à 123.5 & Marnes & 1.31 & 10.5 & 2.87 & 41.8 \\
121.5 à 122.5 & Marnes & 2.00 & 24.4 & 4.80 & 86.8 \\
120.5 à 121.5 & Marnes & 2.80 & 39.1 & 3.76 & 46.1 \\
\hline
\end{tabular}

Les caractéristiques des remblais ont d'abord été estimées à partir de sondages réalisés dans des remblais voisins. Après les travaux de remblaiement et de compactage effectués dans les règles de l'art, deux sondages de contrôle ont révélé que si la compacité du sol était conforme aux prévisions, son module de réaction élastique ne l'était pas : $5 \mathrm{MPa}$ au lieu de 8 MPa. Pour limiter la déflection en tête des pieux, des plots en béton armé de $3 \mathrm{~m}$ de côté et de 1 m d'épaisseur ont été installés autour des pieux, le dessus des plots à la cote 131.0 m IGN69, augmentant ainsi la mobilisation de la réaction élastique du remblai.

\subsection{Choix des caractéristiques des pieux}

La pression exercée par les pieux sur le sol est limitée à Pl/2. Les contraintes appliquées par les pieux sur le sol étant essentiellement dynamiques, le dimensionnement des pieux a été effectué avec des valeurs de la réaction élastique du sol et de la réaction de butée divisées par deux $\left(\right.$ Wilson ${ }^{4}$ ). Dans ces conditions, compte tenu des plots en béton armé, les pieux, en acier de nuance X70, ont un diamètre de $1066 \mathrm{~mm}$, une épaisseur de $18 \mathrm{~mm}$ et une longueur de 21 m. Arasés à la cote $141.2 \mathrm{~m}$ IGN69, leur fiche dans le sol est de $10.8 \mathrm{~m}$.

Les pieux sont disposés en quinconce de part et d'autre des atténuateurs afin d'éliminer les effets d'excentricité en mettant le centre de gravité de l'ASB dans le plan défini par ses pieux, et de limiter les interactions des pieux dans le sol en éloignant deux pieux voisins à une distance supérieure à 3 ou 4 fois leur diamètre.

\subsection{Tolérances de battage}

Les colliers devant glisser sur les pieux sans jeu, il faut utiliser un gabarit de guidage et assurer avec précision le parallélisme des pieux de chaque module durant le battage : 
- le premier pieu est battu avec une tolérance de 2\%o sur sa verticalité ( $2 \mathrm{~cm}$ sur $10 \mathrm{~m}$ ), les tolérances sur la position $X, Y$ étant moins sévères $( \pm 3 \mathrm{~cm})$

- le second pieu est mis en place avec la même tolérance, en maîtrisant au mieux la distance entre les deux pieux. La tolérance sur la position angulaire du second pieu par rapport au premier est plus grande $\pm 3.3 \%$ o ( $\pm 3 \mathrm{~cm}$ sur $9.04 \mathrm{~m}$ ).

\section{Structure des atténuateurs}

\subsection{Technologie et matériaux des caissons}

Les pieux sont intégrés dans la surface de la plaque. Long de $12.0 \mathrm{~m}$, large de $3.0 \mathrm{~m}$ et haut de 2.8 m, l'atténuateur ASB-3000-12 est donc compatible avec le gabarit routier en convoi exceptionnel à autorisation permanente. La couverture par un platelage en bois confère aux atténuateurs un aspect semblable à celui des pontons de plaisance et les rend accostables par les navires de plaisance.

Pour des appareils de petite dimension, il n'est guère possible d'utiliser un autre matériau que l'acier. Le béton armé pourrait représenter une alternative pour les grandes dimensions (au-delà de 5 ou $6 \mathrm{~m}$ ) quand les épaisseurs constructives nécessaires ne sont plus une entrave à la flottabilité de la structure.

Afin d'éliminer les problèmes liés à la corrosion et à l'entretien des aciers peints, les ASB sont construits avec une peau en acier Inox. Ce choix a été assumé dans des conditions économiques raisonnables en séparant, d'une part la fonction de résistance et de raideur de l'ensemble assurée par un treillis en profilés creux rectangulaires galvanisés, et d'autre part la fonction de transmission de la pression du fluide à la structure et d'étanchéité assurée par la peau en tôle d'acier Inox. La disposition de tubes reliant les jupes permet à l'atténuateur de se poser lors des vidanges annuelles du lac.

Pour s'affranchir des risques de gel, le ballast des appareils est constitué de blocs en béton rangés dans des puits destinés à en éviter tout mouvement. Les caissons sont rendus insubmersibles au moyen d'un remplissage en blocs de polystyrène expansé. L'ensemble de la structure a fait l'objet de calculs de structures au moyen d'un modèle aux éléments finis (Robot).

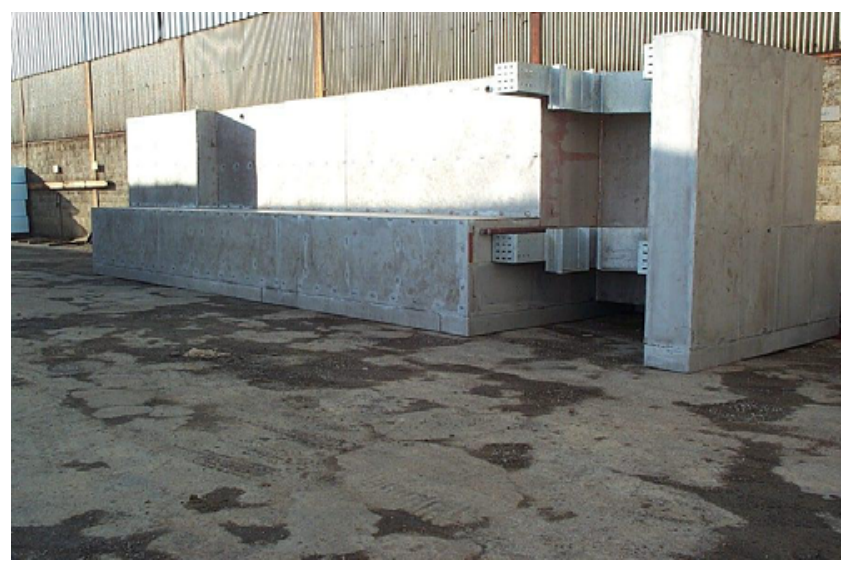

Figure 2: ASB-3000-12: sortie d'usine Degaie à Pont/Sambre

\subsection{Colliers d'ancrage sur les pieux}

La transmission des efforts entre l'atténuateur et les pieux se fait au moyen de colliers en acier galvanisé munis de six patins rectangulaires en polyéthylène UHMW (polyéthylène basse pression à très haut poids moléculaire) supportant une pression normale maximale de 
contact de $30 \mathrm{MPa}$. Hauts de $0.4 \mathrm{~m}$ et larges de $0.3 \mathrm{~m}$ les patins sont usinés pour présenter le même rayon de courbure que le pieu. Dans les cas extrêmes, la contrainte d'appui est de l'ordre de 6.4 MPa pour un seul patin en contact et de 3.2 MPa pour deux patins en contact. Auto lubrifiant à l'eau, le polyéthylène à haute densité a un coefficient de frottement dynamique de 0.1 .

L'efficacité du système nécessite de bloquer au mieux tous les degrés de liberté autres que le pilonnement. Toutefois, compte tenu des tolérances de battage, pour que l'atténuateur puisse glisser librement le long des pieux, les colliers doivent être montés avec un de jeu de l'ordre de 1 à $2 \mathrm{~cm}$ au rayon. Pour éviter les à-coups lors du fonctionnement oscillatoire, les colliers sont munis d'un dispositif anti-chocs permettant aux patins d'être en permanence au contact du pieu et de se s'enfoncer dans leur boîtier sur 2 ou $3 \mathrm{~cm}$ en mobilisant progressivement leur capacité à transmettre l'effort entre l'atténuateur et le pieu.

Enfin, l'effort important, de l'ordre de $800 \mathrm{kN}$, qu'un anneau doit transmettre au pieu a rendu nécessaire un calcul des contraintes de poinçonnement afin d'éviter tout risque de cloquage ou d'ovalisation de la peau du pieu.

\section{Conclusion}

Les différents points techniques abordés dans le cadre de ce projet montrent bien la complexité des problèmes liés à la protection efficace d'un plan d'eau par des systèmes flottants dès que les conditions d'agitation dépassent $1 \mathrm{~m}$ et $4 \mathrm{~s}$. L'utilisation d'atténuateurs semi-submersibles présente un réel avantage lorsque la construction de digues n'est pas possible pour des raisons environnementales, techniques ou financières, ou lorsque les conditions d'agitation imposent des dimensions trop importantes à des solutions flottantes plus classiques, ce qui entraîne un sur dimensionnement des pieux pour obtenir des périodes propres suffisamment basses.

\section{Références}

1.BRLi, Cabinet Fontès, 2000. Etude de définition des aménagements structurants des ports de Nemours et de Nuisement, Rapport BRLi, 180 p.

2.Bougis J. et Degaie A., 1995. L'ASB-5000 : un nouveau concept d'atténuateurs de houles, Actes de Cités Marines 95 - Monaco, pp.349-354.

3.Bougis J., 1995. Analyse des essais en canal des atténuateurs de houle au LNH d'EDF à Chatou, Rapport n94.011, 1000 p.

4.Wilson J.F., 1984. Dynamics of offshore structures, John Wiley, 546 p. 